\title{
Changes in bone mineral density over 18 months following kidney transplantation: the respective roles of prednisone and parathyroid hormone
}

\author{
Jean-Paul Casez ${ }^{1}$, Kurt Lippuner ${ }^{2}$, Fritz F. Horber ${ }^{3}$, André Montandon ${ }^{2}$ and Philippe Jaeger ${ }^{1}$ \\ ${ }^{1}$ Department of Nephrology, University of Nice, France, ${ }^{2}$ Policlinic of Medicine, University of Berne and ${ }^{3}$ Klinik \\ Hirslanden, Zürich, Switzerland
}

\begin{abstract}
Background. Prednisone is a major factor of bone loss after kidney transplantation. The role of hyperparathyroidism and immunosuppressors is less clear.

Methods. Thirty-three patients (14 men, 19 women) with ESRD were followed prospectively for 18 months after kidney transplantation. All patients received prednisone and cyclosporin A (CyA) with $(n=18)$ or without azathioprine. Rejection episodes were treated with boluses of methylprednisolone. Bone mineral density (BMD) was measured using dual-energy X-ray absorptiometry for the spine, hip and whole body (total, trunk, limbs) at 1, 12, 24, 36, 60 and 75 weeks after kidney transplantation. At the same time, blood was assayed for calcium, phosphorus, intact-PTH, alkaline phosphatase, creatinine and CyA, and 24-h urine was assayed for $\mathrm{Ca}$ and $\mathrm{P}$.

Results. BMD at baseline was low at all skeletal sites in women, but not in men. BMD decreased significantly at the spine $(-7.0 \pm 0.9 \%$, week 24$)$, trunk $(-4.8 \pm 0.5 \%$, week 24$)$, total hip $(-4.3 \pm 1.0 \%$, week 36), whole body $(-2.2 \pm 0.4 \%$, week 36$)$ and limbs $(-1.0 \pm 0.7 \%$, week 74$)$. BMD changes over time followed three different patterns: no change or gain, continuous loss, and NADIR. For the spine and trunk, two thirds of patients had a NADIR pattern with recovery at the end of the study, and one-quarter of patients had continuous bone loss. For the limbs, BMD rose or remained stable $(n=20)$, decreased continuously $(n=8)$ or had a NADIR pattern $(n=5)$. Neither gender nor time on dialysis prior to transplantation influenced BMD changes. Patients with PTH serum concentrations below the median value 1 week after kidney transplantation $(109 \mathrm{pg} / \mathrm{ml})$ had continuous bone loss at the whole body or limbs but not at other sites. The cumulative dose of prednisone correlated negatively with BMD changes at the spine
\end{abstract}

Correspondence and offprint requests to: Prof. Philippe Jaeger, Department of Nephrology, University Hospital, PO Box 69, F-06002 Nice Cedex 1, France. Email: philippe.jaeger@freesurf.ch $(r=-0.39, P<0.03)$, hip $(r=-0.50, P=0.005)$ and trunk $(r=-0.52, P=0.002)$, but not at the whole body or limbs. CyA levels in blood did not correlate with BMD changes. BMD for the whole body and limbs did not change in the patients receiving azathioprine $(n=16 ;-2.7 \pm 0.7 \%, P=0.013)$ but decreased in the others $(-2.8 \pm 0.9 \%, P<0.0002)$.

Conclusions. High cumulative prednisone doses are deleterious for the axial skeleton. Low levels of PTH observed 1 week after kidney transplantation are predictive of continuous cortical bone loss.

Keywords: azathioprine; bone mineral density; cyclosporin A; hyperparathyroidism; prednisone; renal transplantation

\section{Introduction}

It has been shown repeatedly that bone mineral density (BMD) decreases significantly within the first 6 months after kidney graft in patients with end-stage renal disease, mainly due to glucocorticoid therapy [1,2]. Long-term stabilization and even recovery of bone mass after kidney grafting has been observed and may depend on several factors, including gender, skeletal site and parathyroid status [1,3]. From animal studies it is known that cyclosporin may also influence bone density either positively $[4,5]$ or negatively [6].

Because the respective roles of hyperparathyroidism and immunosuppressive treatment at various postoperative time points remain controversial, we have studied prospectively 33 renal transplant patients for 18 months after grafting.

\section{Patients and methods}

\section{Patients and normal subjects}

Of 47 renal transplant patients enrolled into the study, 14 dropped out for various reasons (four died, one had 
irreversible graft rejection, one had iterative rejection crises, three refused to complete the study and five moved out of town). Thirty-three patients completed the 18 months of the study, of whom 14 were men and 19 women (10 premenopausal, 9 post-menopausal, none of them receiving hormone replacement therapy). Age at inclusion (mean \pm SEM) was $46 \pm 2$ years and body mass index (BMI) was $22.0 \pm 0.6 \mathrm{~kg} / \mathrm{m}^{2}$ (males $23.8 \pm 0.9$, females $20.7 \pm 0.7$ ). End-stage renal failure was due to glomerulonephritis $(n=12)$, analgesic nephropathy $(n=5)$, reflux nephropathy $(n=4)$, polycystic kidney disease $(n=8)$, chronic pyelonephritis $(n=2)$, nephroangiosclerosis $(n=1)$ or Fabry's disease $(n=1)$. Before kidney grafting patients had been on chronic dialysis (haemodialysis, $n=30$, and chronic ambulatory peritoneal dialysis, $n=4$ ) for $63 \pm 12$ months (range 2-303).

The study was approved by the ethical committee at the University of Berne School of Medicine and informed consent was obtained from all subjects after the nature of the procedures had been fully explained.

\section{Study design}

Patients were investigated at weeks 1, 6, 12, 24, 36, 60 and 75 after kidney transplantation, and underwent dual-energy X-ray absorptiometry (DXA) measurements, fasting blood sampling and 24-h urine collection. Bone biopsies had been planned initially, but then were cancelled from the protocol due to major complaints related to pain and haematoma in the few patients who underwent this procedure.

\section{Immunosuppression and concomitant drug therapy}

All patients were treated with prednisone (Figure 1) and cyclosporin A (CyA) given at the standard dosage (see below). One patient with tuberculosis, however, received very high doses of CyA as a consequence of rifampicin therapy. Azathioprine was introduced in 11 patients at the time of transplantation and in seven patients later on.

Acute graft rejection was treated with intravenous methylprednisolone $500 \mathrm{mg} / \mathrm{day}$, for a maximum of 6 days
(13 rejection episodes in 12 of the 33 patients who completed the study). Due to symptomatic hypophosphataemia, 10 patients received phosphate supplements. None of the patients received vitamin $\mathrm{D}$, oestrogen replacement therapy or treatment against osteoporosis. Hypertension $(n=29)$ was treated with beta-blockers and/or calcium channel blockers. Ranitidine or omeprazole were used to prevent peptic ulcer disease in all patients when the daily dose of prednisone was $>15 \mathrm{mg}$.

\section{Dual-energy X-ray absorptiometry}

DXA of whole body (at all visits), lumbar spine and hip (at all visits except on week 6) was performed using Hologic ${ }^{\mathbb{R}}$ scanners (QDR 1000W or QDR 2000), with each patient being followed on the same machine throughout the study. Precision error was $0.4 \%$ for BMD, based on daily scanning of an anthropomorphic phantom supplied by the manufacturer. No drift was seen during the period of investigation. Based on repeated measurements performed at our institution, precision error in vivo is $1 \%$ (i.e. $0.01 \mathrm{~g} / \mathrm{cm}^{2}$ ) or less for all sites; therefore a difference of $>0.028 \mathrm{~g} / \mathrm{cm}^{2}$ between two scans was considered significant.

Analysis was performed according to standard procedures for the spine, hip and whole body. For whole body analysis, a subanalysis of the trunk (i.e. spine and pelvis) and limbs (i.e. both arms and both legs) was carried out to separate axial from appendicular skeleton, respectively. Results at baseline were expressed as BMD $\left(\mathrm{g} / \mathrm{cm}^{2}\right)$ for all bone sites and also as $Z$-scores (standard deviation from the mean value of age- and sex-matched normal controls from the area of Berne) for lumbar spine and femoral neck.

\section{Blood and urine collection}

Blood was drawn after an overnight fast and 24-h urine was collected for analysis. Plasma concentrations of total calcium, phosphorus and creatinine, total plasma alkaline phosphatase activities, as well as urinary concentrations of calcium and creatinine were analysed using standard techniques in the central laboratory at our institution. The

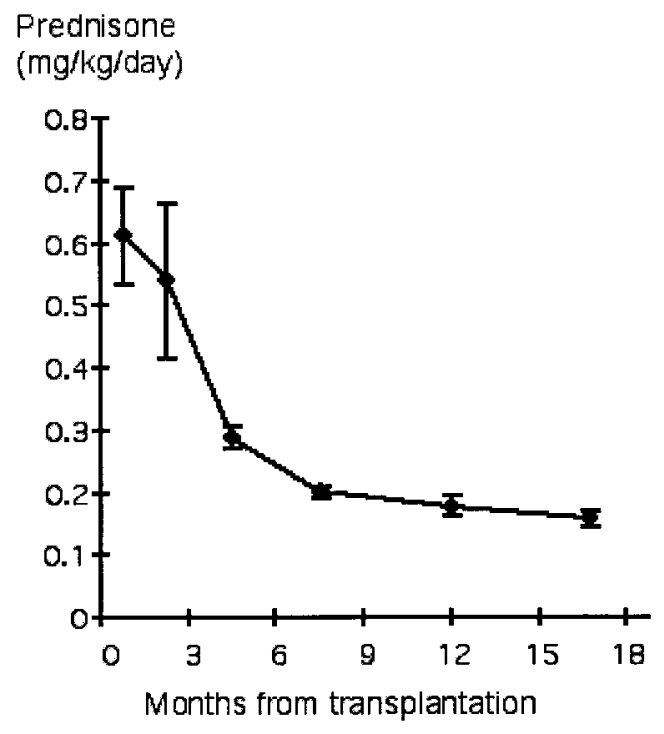

\section{Prednisone}

(mg/kg)

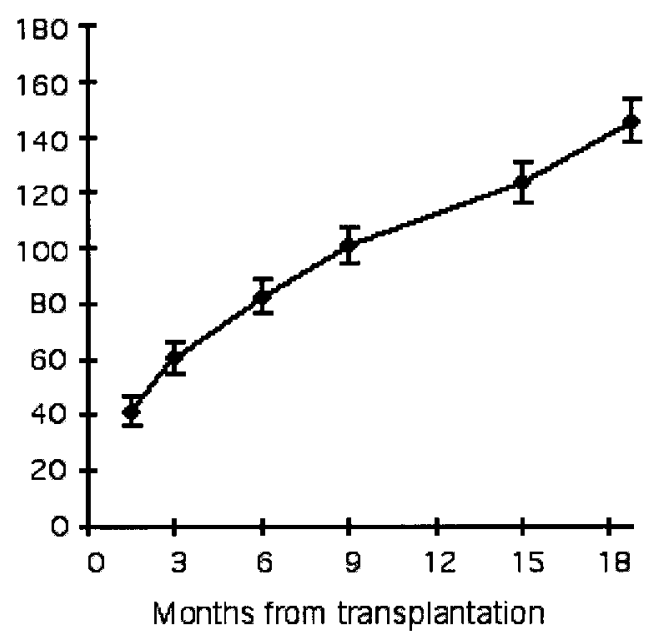

Fig. 1. Daily and cumulative doses of prednisone (mean \pm SEM) administered after kidney transplantation in 33 patients. 
serum concentration of intact parathyroid hormone (PTH) was assayed using the Allegro kit (Nichols Institute, San Juan Capistrano, CA, USA). PTH values at baseline were classified according to the median value into two groups, namely low PTH and high PTH. CyA trough blood levels were measured.

In addition, results were noted a posteriori for the last serum PTH measurement performed prior to transplantation.

\section{Statistics}

Statistics (one-way and two-way analysis of variance (ANOVA) for repeated measures, simple regression analysis and unpaired $t$-tests) were performed using Statview ${ }^{\mathbb{R}}$ 4.5 (Abacus Concept, Berkeley, CA, USA) on a Macintosh computer. $P<0.05$ was considered significant. To normalize the distribution, decimal log transformation was applied to PTH results.

\section{Results}

\section{Immunosuppressive therapy}

The cumulative prednisone dose (expressed as $\mathrm{mg} / \mathrm{kg}$ body weight) and the mean daily dose since the last visit ( $\mathrm{mg} /$ day/kg body weight) were calculated at each visit and are shown in Figure 1: a three-fold reduction in the daily dose was achieved between week 4 $(0.6 \mathrm{mg} / \mathrm{kg})$ and week $30(0.2 \mathrm{mg} / \mathrm{kg})$, followed by a slower decrement until week 44, the dose being kept stable thereafter $(0.16 \mathrm{mg} / \mathrm{kg})$. The prednisone dose per kilogram of body weight did not differ between men and women.

Cyclosporin trough levels (Table 1) decreased from week $1(482 \pm 27 \mathrm{ng} / \mathrm{ml})$ to week $24(313 \pm 17 \mathrm{ng} / \mathrm{ml})$, then remained stable without differences between males and females.

\section{Biochemical parameters (Table 1)}

Immediately after kidney transplantation, mean total plasma calcium concentration was normal. However, mean albumin-corrected calcium concentration was slightly above the normal range because serum albumin levels were low. The mean \pm SEM of parathyroid hormone serum levels was $140 \pm 24 \mathrm{pg} / \mathrm{ml}$, with a median value of $109 \mathrm{pg} / \mathrm{ml}$ defining the high PTH and the low PTH groups, far above the upper limit of normal range $(65 \mathrm{pg} / \mathrm{ml}$; lowest and highest quartile thresholds were $51 \mathrm{pg} / \mathrm{ml}$ and $174 \mathrm{pg} / \mathrm{ml}$, respectively). Mean plasma phosphate concentration was below the normal range and mean plasma alkaline phosphatase activity was within it. Creatinine clearance was low, as was 24-h urinary excretion of calcium. There was no significant difference between genders for any parameter at baseline (data not shown).

At follow-up, mean total plasma calcium concentration rose until week 12 to reach the upper limit of the normal range, then remained stable. Mean serum albumin level increased until week 36 to reach normal

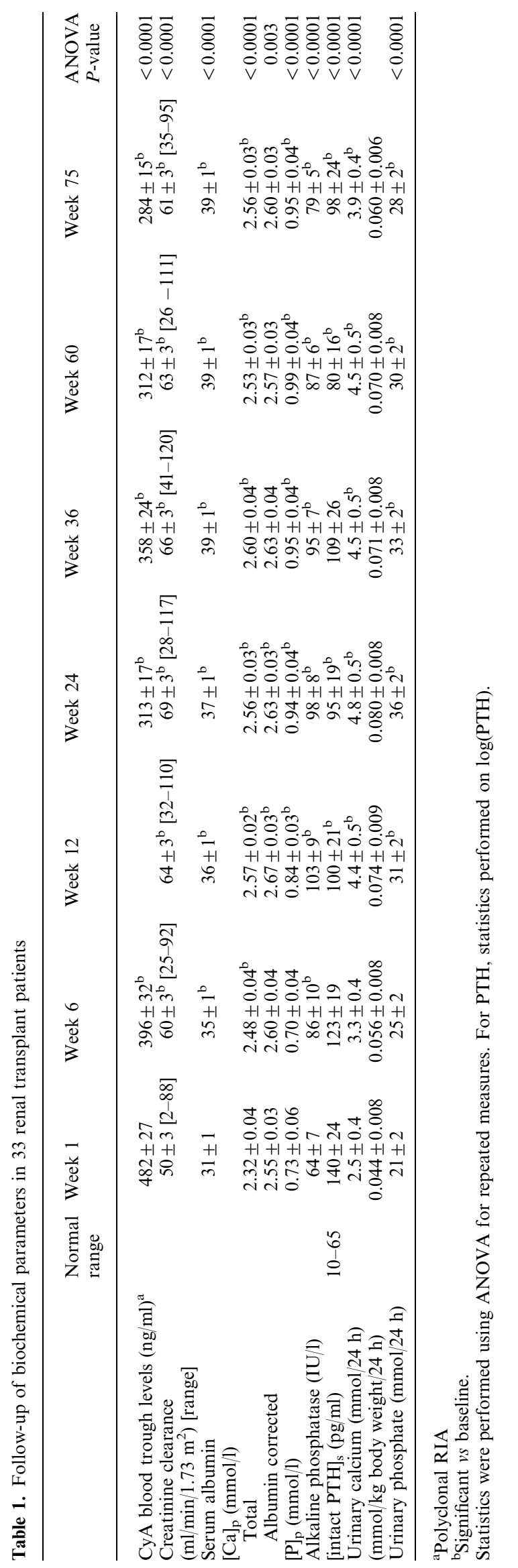


values, then remained constant. Albumin-corrected calcium concentration peaked at week $12(2.67 \mathrm{mmol} / \mathrm{l})$, then returned to the upper normal range. Intact PTH serum levels decreased continuously until week 60 , but always remained above the normal range. Serum phosphate concentrations remained low during the first 6 weeks after grafting, then increased continuously up to normal values until week 60. Mean alkaline phosphatase activity peaked at week 12 (upper limit of the normal range) then decreased continuously until the end of the study period. Creatinine clearance rose to close to the low normal range at week 24 , then decreased slightly. Urinary calcium $(24 \mathrm{~h})$ increased from low to normal after 12 weeks.

When all time points were taken into account, men had slightly higher creatinine clearance and 24-h calciuria than women (data not shown). Gender did not influence the remaining parameters.

Because many patients were followed outside our institution during the dialysis period, where other types of PTH assay were performed (C-terminal, $\mathrm{N}$-terminal, mid-molecule), valid intact $\mathrm{PTH}$ results prior to transplantation could be retrieved in only 10 patients. The mean concentration was $171 \pm 50 \mathrm{pg} / \mathrm{ml}$, not significantly higher than the mean value observed immediately after kidney transplantation $(112 \pm 31 \mathrm{pg} / \mathrm{ml})$. There was no correlation between serum intact PTH levels before and immediately following transplantation.

\section{Bone mineral density}

Baseline values are shown in Table 2: at all bone sites, women had low BMD compared with age-matched healthy controls ( $Z$-score), whereas men had normal BMD. There was no significant difference in BMD

Table 2. Bone mineral density at baseline in 33 kidney transplant patients

\begin{tabular}{|c|c|c|c|}
\hline & $\begin{array}{l}\text { Females } \\
(n=19)\end{array}$ & $\begin{array}{l}\text { Males } \\
(n=14)\end{array}$ & $\begin{array}{l}P \text {-value } \\
\text { (males } v s \text { females) }\end{array}$ \\
\hline \multicolumn{4}{|l|}{ L2-L4 } \\
\hline BMD & $0.889 \pm 0.031$ & $1.007 \pm 0.037$ & 0.02 \\
\hline$Z$ score & $-0.87 \pm 0.24^{\mathrm{a}}$ & $-0.10 \pm 0.28$ & $<0.05$ \\
\hline \multicolumn{4}{|c|}{ Femoral neck } \\
\hline BMD & $0.641 \pm 0.025$ & $0.791 \pm 0.050$ & $<0.01$ \\
\hline$Z$ score & $-1.3 \pm 0.2^{\mathrm{a}}$ & $-0.2 \pm 0.4$ & $<0.02$ \\
\hline \multicolumn{4}{|l|}{ Total hip } \\
\hline BMD & $0.697 \pm 0.029$ & $0.873 \pm 0.042$ & 0.001 \\
\hline$Z$ score & - & - & \\
\hline \multicolumn{4}{|c|}{ Whole body } \\
\hline BMD & $0.937 \pm 0.024$ & $1.114 \pm 0.032$ & 0.0001 \\
\hline$Z$ score & $-1.3 \pm 0.3^{\mathrm{a}}$ & - & - \\
\hline \multicolumn{4}{|l|}{ Trunk } \\
\hline BMD & $0.862 \pm 0.030$ & $1.014 \pm 0.045$ & 0.01 \\
\hline$Z$ score & - & - & \\
\hline \multicolumn{4}{|l|}{ Limbs } \\
\hline BMD & $0.885 \pm 0.021$ & $1.100 \pm 0.038$ & 0.0001 \\
\hline$Z$ score & - & - & \\
\hline
\end{tabular}

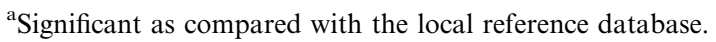

Statistics performed using Student's $t$-test. (absolute values or $Z$-score) according to baseline PTH levels (data not shown).

BMD changes over time are depicted in Table 3. At all sites BMD decreased significantly, the decrement being more marked at the spine $(-7.0 \pm 0.9 \%$, week 24), trunk ( $-4.8 \pm 0.5 \%$, week 24$)$ and total hip $(-4.3 \pm 1.0 \%$, week 36$)$ than at the whole body $(-2.2 \pm 0.4 \%$, week 36$)$ and limbs $(-1.0 \pm 0.7 \%$, week 74$)$.

Individual analysis of BMD follow-up led to detection of the three different patterns summarized in Table 4: no change or gain, continuous loss, and NADIR. At the spine and trunk, which contain significant amounts of trabecular bone, approximately one-quarter of the patients continuously lost bone mineral throughout the study, whereas approximately two-thirds of them had a NADIR pattern, i.e. a significant bone mineral loss $\left(>0.028 \mathrm{~g} / \mathrm{cm}^{2}\right)$ followed by a significant bone mineral gain $\left(>0.028 \mathrm{~g} / \mathrm{cm}^{2}\right)$, the NADIR occurring around 6 months after grafting. At limbs that consist of almost pure cortical bone, one-quarter of patients also continuously lost bone throughout the study, whereas most patients had no significant change or even gained bone mineral. At the hip and whole body (mixed cortical and trabecular bone), an intermediate distribution of the three patterns was observed. Figure 2 illustrates the mean changes at the limbs and trunk according to these three different patterns.

\section{Factors governing BMD changes over time}

Gender or time on dialysis before transplantation did not influence BMD changes at any site. Such was also the case for menopausal status in women.

Body mass index. There was no significant correlation between changes in BMD of lumbar spine or hip and changes in BMI at any corresponding time point. There was, however, a negative correlation between BMD changes in whole body or limbs and BMI changes at week $12(r=0.69, P<0.0001$, and $r=0.54, P=0.001$, respectively), week $24(r=0.65$, $P<0.0001$, and $r=0.67, P=0.0001$, respectively) and week $36(r=0.52, P=0.002$, and $r=0.46, P=0.01$, respectively), but there was no longer a correlation at week 64 . Conversely for the trunk, a positive correlation between changes in BMD and BMI was found exclusively at week 64. There was no correlation between BMI changes and the cumulative dose of prednisone at corresponding timepoints or with PTH level at baseline.

Parathyroid status. Regression analysis of BMD changes from baseline vs PTH levels (log transformed) at baseline revealed positive correlations at the whole body (week 60: $r=0.39, P<0.03$; week 75: $r=0.48, \quad P<0.01$ ), the trunk (week 75: $r=0.36$, $P=0.04$ ) and the limbs (week 6: $r=0.36, P=0.04$; week 60: $\quad r=0.40, \quad P<0.02$; week 75: $r=0.48$, $P<0.01$ ). Similar correlations (data not shown) were also found between BMD changes and PTH serum 


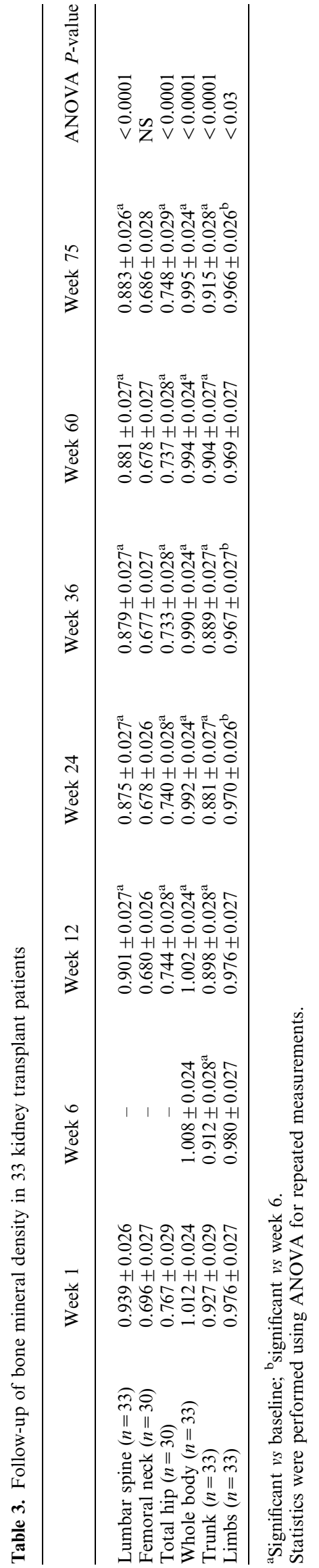

levels for the whole body, trunk or limbs at week 6, but not thererafter. There was no correlation between PTH levels and BMD changes at the lumbar spine or hip.

Figure 3 depicts BMD changes as a function of time after grafting analysed according to PTH levels at baseline. The low PTH group displayed a continuous loss, reaching $\sim 3 \%$ after 18 months in the whole body and the limbs (both $P<0.001$ ). In contrast in the high PTH group, BMD remained constant in the limbs and showed a nadir pattern with complete recovery in the whole body. Conversely, parathyroid status at baseline did not significantly influence BMD changes at the lumbar spine, hip or trunk.

Prednisone. There was a negative correlation between the cumulative prednisone dose after 18 months and changes in BMD over the same period of time at the spine $(r=0.39, P<0.03)$, the hip $(r=0.50$, $P=0.005)$ and the trunk $(r=0.52, P=0.002)$, but not in the whole body or limbs. There was no correlation between BMD recovery and the mean daily or cumulative prednisone dose administered after the nadir.

$C y A$. No correlation was found between BMD changes at any site and CyA blood trough levels measured at any time.

Azathioprine. Whole-body and limb BMDs did not change over time in patients receiving azathioprine $(n=16,-0.5 \pm 0.7 \%$ and $1.0 \pm 0.9 \%$, respectively), whereas they decreased significantly in patients not receiving that drug $(n=17 ;-2.7 \pm 0.7 \%, P=0.013$ and $-2.8 \pm 0.9 \%, P<0.0002$, respectively), although there was no difference in terms of cumulative prednisone dose or PTH serum levels between groups. However, this apparent protective effect of azathioprine on the skeleton was not noticed at the spine, hip or trunk.

\section{Discussion}

This prospective study carried out in 33 patients followed for 18 months after kidney transplantation reveals that: (i) prednisone induces a dose-dependent bone loss in the axial skeleton, which is recoverable in most patients; and (ii) the parathyroid hormone level at the time of transplantation is an indicator of bone loss in limbs.

It has been clearly established that axial bone loss occurs following renal transplantation. Julian et al. [1] first demonstrated in 20 kidney recipients a $6.8 \%$ and $8.8 \%$ decrease in lumbar bone density 6 and 18 months after transplantation, respectively, and concluded that prednisone mainly accounted for vertebral bone loss. Similar results obtained over 5 months after transplantation have been published by our group [2]. The present study of 33 patients followed for 18 months confirms the prednisone-induced bone loss in the axial skeleton with clear dose dependency at the lumbar 
Table 4. Patterns of bone mineral density follow-up in 33 kidney transplant patients

\begin{tabular}{|c|c|c|c|c|c|}
\hline BMD profile & $\begin{array}{l}\text { Lumbar spine } \\
(n=33)\end{array}$ & $\begin{array}{l}\text { Total hip } \\
(n=30)\end{array}$ & $\begin{array}{l}\text { Whole body } \\
(n=33)\end{array}$ & $\begin{array}{l}\text { Trunk } \\
(n=33)\end{array}$ & $\begin{array}{l}\text { Limbs } \\
(n=33)\end{array}$ \\
\hline Gain or no change $^{\mathrm{a}}$ (No. of patients) & 2 & 12 & 13 & 6 & 20 \\
\hline Continuous $\operatorname{loss}^{\mathrm{a}}$ (No. of patients) & 8 & 11 & 12 & 8 & 8 \\
\hline Total loss $\left(\mathrm{g} / \mathrm{cm}^{2}\right)$ & $-0.108 \pm 0.018$ & $-0.084 \pm 0.010$ & $-0.049 \pm 0.007$ & $-0.077 \pm 0.009$ & $-0.067 \pm 0.012$ \\
\hline NADIR $^{\mathrm{a}}$ (No. of patients) & 23 & 7 & 8 & 19 & 5 \\
\hline Loss at nadir $\left(\mathrm{g} / \mathrm{cm}^{2}\right)$ & $-0.079 \pm 0.007$ & $-0.062 \pm 0.007$ & $-0.049 \pm 0.009$ & $-0.062 \pm 0.004$ & $-0.040 \pm 0.005$ \\
\hline Gain post nadir $\left(\mathrm{g} / \mathrm{cm}^{2}\right)$ & $+0.083 \pm 0.007$ & $+0.059 \pm 0.010$ & $0.041 \pm 0.004$ & $+0.067 \pm 0.006$ & $+0.048 \pm 0.008$ \\
\hline \multicolumn{6}{|l|}{ Time at nadir } \\
\hline Week 6 & - & - & 2 & 0 & 1 \\
\hline Week 12 & 3 & 1 & 1 & 6 & 1 \\
\hline Week 24 & 11 & 2 & 3 & 8 & 1 \\
\hline Week 36 & 9 & 3 & 1 & 4 & 1 \\
\hline Week 64 & 0 & 1 & 1 & 1 & 1 \\
\hline
\end{tabular}

${ }^{\mathrm{a}}$ Based on precision error of $0.01 \mathrm{~g} / \mathrm{cm}^{2}$, a difference in BMD of $>0.028 \mathrm{~g} / \mathrm{cm}^{2}$ between two measurements was considered as significant $(P<0.05)$.

\section{(\% from baseline) $\quad$ TRUNK}

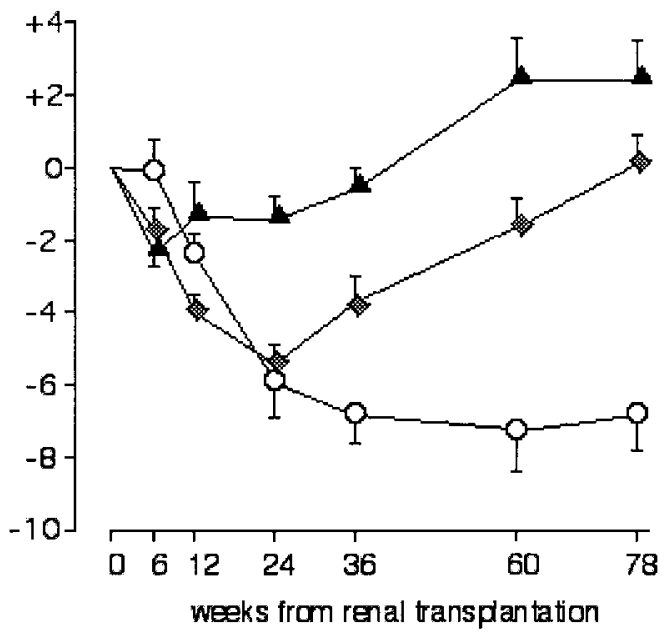

\section{(\% from baseline)}

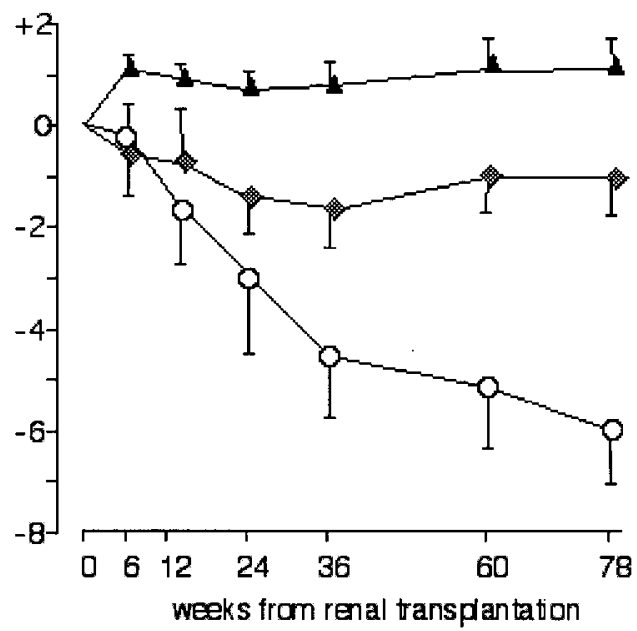

$\Delta$ no change or bone gain $(n=6)$

\section{NADIR pattern (n=19)}

O Continuous bone loss (n=8)

Fig. 2. BMD changes in the trunk and limbs after kidney transplantation according to follow-up pattern. $P<0.0001$ between patterns (two-way ANOVA for repeated measurements).

spine, hip and trunk, in agreement with findings by other groups $[7,8]$. Such a marked effect on axial skeleton that contains most of the trabecular bone was expected, analogous to the well-known glucocorticosteroid-induced osteoporosis. It must be pointed out, however, that a few patients lost no bone mineral at all.

The reversibility of bone loss after grafting has been already suggested by Almond et al. [3], who observed some skeletal recovery after 6 to 12 months in the femoral neck and whole skeleton. In the present study, Figure 2 and Table 4 illustrate that according to their BMD changes, patients can be classified in three categories: no change or gain, nadir pattern with recovery, and continuous loss. BMD increased in two-thirds of the patients in the lumbar spine and trunk after a nadir found at $\sim 6$ months posttransplantation. In general, a complete BMD recovery was observed in the trunk, which contrasted with the marginal recovery found in the lumbar spine. In fact, this apparent stagnation of lumbar BMD after 6 months is due to a continuous bone loss observed in eight of the 33 patients, a phenomenon that masked the complete recovery observed in those 23 patients with the nadir pattern (Table 4). It is noteworthy that continuous bone loss in the trunk was associated with a higher cumulative prednisone dose $(185 \pm 19$ vs $133 \pm 7 \mathrm{mg} / \mathrm{kg} ; P<0.01$ ), higher daily prednisone dose throughout the study $(P<0.01)$, and more boluses of methylprednisolone to control rejection episodes. This underlines the fact that repeated and very high doses of glucocorticosteroid may inhibit bone recovery for a long time.

Whole body skeleton, which is made of up to $80 \%$ cortical bone, and limb skeleton, which is almost 
BMD

(\%trom baseline)

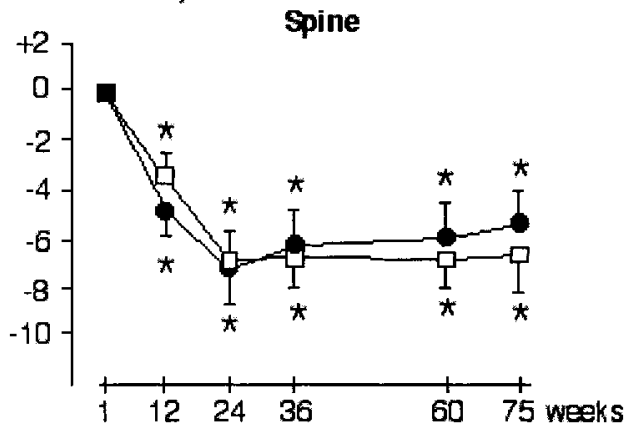

BMD

(\%trom baseline)

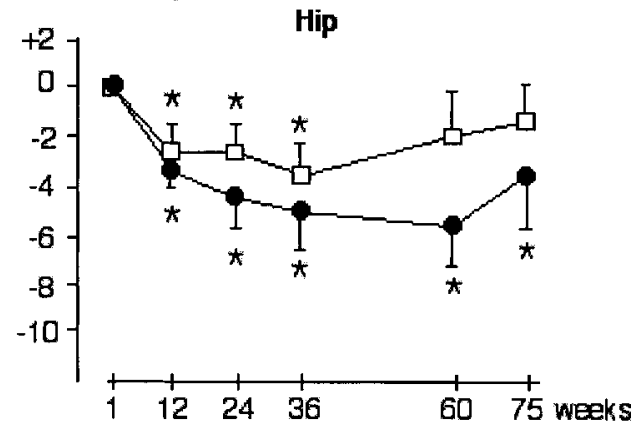

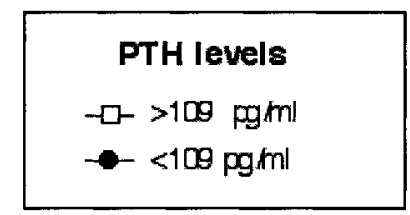

Trunk

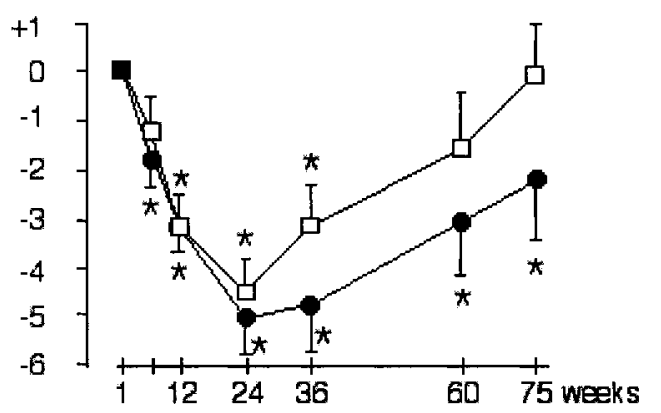

Whole Body

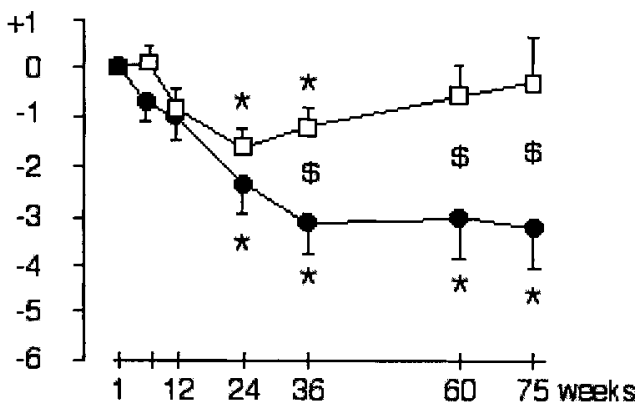

Limbs

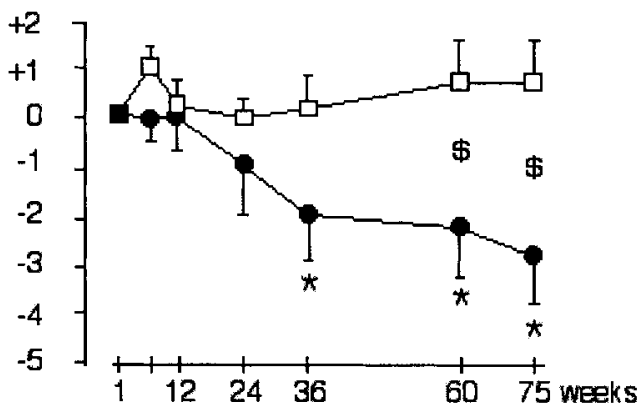

Fig. 3. BMD changes in the spine, total hip, whole body, trunk and limbs following kidney transplantation in 33 patients, according to serum intact PTH 1 week after grafting. In the whole body and limbs, the level of serum PTH has a highly significant effect on BMD changes $\left(P<0.0001\right.$, two-way ANOVA for repeated measurements). *Significant $v s$ baseline. ${ }^{\$}$ Significant between groups.

entirely made of cortical bone, were not significantly influenced by the dose of prednisone. In contrast, the baseline PTH level was a major determinant of BMD changes at these 'cortical' sites. Indeed, patients with baseline PTH levels below the median value (i.e. $109 \mathrm{pg} / \mathrm{ml}$ ) displayed continuous mineral loss after grafting, in contrast to patients whose basal PTH levels were $>109 \mathrm{pg} / \mathrm{ml}$. There was no difference in BMD at any bone site at the time of transplantation between the two PTH groups and we did not find any correlation between changes in PTH levels and BMD changes over time. It is most likely that a low baseline
PTH level corresponds to renal adynamic bone lesion in many patients. Unfortunately, we were unable to obtain bone biopsies from our patients to verify this hypothesis. From matching to bone biopsies it has been established that low concentrations of serum intact-PTH are highly predictive for a dynamic bone lesion $[9,10]$ for levels ranging, depending on the assay, from onefold [11] to twofold [12] the upper limit of the normal range, which corresponds to $65-130 \mathrm{pg} / \mathrm{ml}$ in the present study. Indeed, some intact-PTH assays not only measure true 1-84 PTH but also one particular C-terminal fragment [13]. However, prediction of 
bone histology from PTH levels has been studied on dialysis, not following transplantation. Indeed, several factors influence PTH concentration 1 week post-graft, including a drop in phosphataemia and acute tubular necrosis. Anyway, our median value of serum PTH concentration 1 week after transplantation $(109 \mathrm{pg} / \mathrm{ml})$ allows the separation of two populations that behave differently in terms of bone density changes. Low PTH levels are associated with continuous cortical bone loss after grafting: osteoblasts receiving no anabolic stimulation by the PTH may be more vulnerable to prednisone [14]. Some 'protective effect' of PTH was also found in the axial skeleton, since patients with continuous loss in the trunk had lower baseline PTH serum levels than patients with nadir, unchanged BMD or bone gain pattern $(63 \pm 13 v s$ $165 \pm 30 \mathrm{pg} / \mathrm{ml}$, respectively; $P=0.02$ ).

Data in the literature are controversial in this respect: Julian et al. [1] did not find a significant correlation between PTH levels at time of transplantation and lumbar bone loss, and Kim et al. [15] obtained similar results. Other studies were consistent with the classical deleterious effect of hyperparathyroidism on bone: Grotz et al. [16] observed that patients with BMD loss in the lumbar spine 1 year after renal transplantation had higher PTH levels at time of transplantation than subjects with stable lumbar BMD. Torregrosa et al. [17] reported that the decrease in BMD during the first 6 months following transplantation was more severe in patients with serum intact-PTH levels $>240 \mathrm{pg} / \mathrm{ml}$ at time of transplantation. Torres et al. [25] also found a negative correlation between changes in vertebral mineral density measured by QCT and PTH levels prior to transplantation. In a study by Almond et al. [3], PTH serum concentrations correlated negatively with lumbar BMD changes in women, and positively with femoral BMD changes in men.

These discrepancies may be related to differences in the severity of secondary hyperparathyroidism between the studies: mean values reported by Julian et al. [1] were similar to those found in the present study. In contrast, in the study by Almond et al. [3], eight of 14 women had very high serum PTH concentrations, $>400 \mathrm{pg} / \mathrm{ml}$, compared with one out of 33 in the present study. Altogether, these data suggest that either low PTH serum concentrations or severe hyperparathyroidism are deleterious to bone.

Unexpected was the sparing effect of azathioprine on cortical skeleton. BMD in the whole body and limbs remained stable over the entire follow-up period in patients receiving azathioprine and decreased in patients not receiving the drug. This occurred independently of the PTH level, prednisone dose and creatinine clearance, which was similar in patients with or without azathioprine. Literature does not provide information on any modulatory role of azathioprine on the human skeleton. In rats, azathioprine appears to have little effect on bone. In azathioprine-treated rats for instance, Bryer et al. [18] observed slightly decreased osteocalcin levels but no difference in trabecular volume, mineral apposition rate, bone formation rate or longitudinal bone growth as compared with controls. In addition, azathioprine failed to alter the high-turnover osteopenia induced by CyA. Thus, the apparent favourable effect of azathioprine on human cortical bone has to be confirmed by further studies.

We did not find an impact of CyA treatment on bone density, i.e. no correlation between BMD changes at any site and blood CyA levels. However, alkaline phosphatase activity rose substantially after grafting, probably due to treatment with CyA as suggested by others [19]. Whether CyA treatment is beneficial or deleterious to bone is a controversial matter and is discussed in the literature [20]. Studies in humans indicate rather beneficial effects. Kelly et al. [21] reported the potential protection afforded by CyA against glucocorticoid effects on bone. In a cross-sectional study, Grotz et al. [7] found a significant positive correlation between the dose of CyA and BMD at the femoral neck but not in the spine. In a prospective study, McIntyre et al. [8] reported a beneficial effect of CyA on distal forearm BMD in 10 patients on CyA monotherapy compared with patients on azathioprine-prednisone, the benefit becoming significant only after the third year. Animal or in vitro studies are more conflicting. Schlosberg and co-workers [6] found evidence that CyA induces severe osteopenia in normal rats, whereas others found no effect or even reduced bone resorption under similar experimental conditions [4,5]. In vitro, CyA inhibits bone resorption induced by parathyroid hormone and calcitriol [22]. Finally, the effect of CyA on bone may be highly dependent on experimental conditions, as underlined by Epstein [20].

The length of the dialysis period prior to transplantation did not influence either BMD at baseline or BMD changes throughout the study. Although BMD changes were similar in both genders throughout the study, women but not men displayed a low BMD $v s$ controls at baseline. The reason for this difference that has been observed earlier [23] remains unclear: hypogonadism consistently occurs in both men and women with terminal renal failure [24]. One might thus presume that the female skeleton is more susceptible to decreased gonadal hormone levels than the male one.

Although the present study has limitations due to a relatively small and heteregeneous sample, it leads to the reasonable conclusion that bone density changes following kidney transplantation may vary from patient to patient: bone density remains stable in some patients, decreases continuously in others, or, which was the case in the majority of our patients, initially decreases and later recovers (at least partially). The type of pattern depends on the skeletal site and is influenced by both the cumulative prednisone dose and the parathyroid status at time of transplantation. Indeed, in our patients, low baseline PTH levels were associated with continuous cortical bone loss after transplantation. Further studies are required to clarify 
the role of CyA and azathioprine on bone after transplantation. Because women but not men display low bone density before transplantation, efforts should be made to prevent osteoporosis in women on dialysis.

Acknowledgements. We are grateful to Mrs Elizabeth Dijkhuis for the collection of data and management of patient charts.

\section{References}

1. Julian B, Laskow D, Dubovsky J, Dubovsky E, Curtis J, Quarles L. Rapid loss of vertebral mineral density after renal transplantation. $N$ Engl J Med 1991; 325: 544-550

2. Horber F, Casez J, Steiger U, Czerniak A, Montandon A, Jaeger P. Changes in bone mass early after kidney transplantation. J Bone Miner Res 1994; 9: 1-9

3. Almond M, Kwan J, Evans K, Cunningham J. Loss of regional bone mineral density in the first 12 months following renal transplantation. Nephron 1994; 66: 52-57

4. Del Pozo E, Lippuner K, Ruch W et al. Different effects of cyclosporine $\mathrm{A}$ on bone remodeling in young and adult rats. Bone 1995; 16: 271S-275S

5. Orcel P, Bielakoff J, Modrowsky D, Miravet L, de Vernejoul M. Cyclosporin A induced in vivo inhibition of resorption and stimulation of formation in rat bone. J Bone Miner Res 1989; 4: $387-391$

6. Schlosberg M, Movsowitz C, Epstein S, Ismail F, Fallon M, Thomas S. The effect of cyclosporin A administration and its withdrawal on bone mineral metabolism in the rat. Endocrinology 1989; 124: 2179-2184

7. Grotz W, Mundinger F, Gugel B, Exner V, Kirste G, Schollmeyer P. Bone mineral density after kidney transplantation, a cross-sectional study in 190 graft recipients up to 20 years after transplantation. Transplantation 1995; 59: 982-986

8. McIntyre H, Menzies B, Rigby R, Perry-Keene D, Hawley C, Hardie I. Long-term bone loss after renal transplantation: comparison of immunosuppressive regimens. Clin Transplantation 1995; 9: 20-24

9. Cohen Solal M, Sebert J, Boudaillez B et al. Comparison of intact, midregion, and carboxy terminal assays of parathyroid hormone for the diagnosis of bone disease in hemodialyzed patients. J Clin Endocrinol Metab 1991; 73: 516-524

10. Fournier R, Oprisiu S, Said A et al. Invasive versus non-invasive diagnosis of renal bone disease. Curr Opin Nephrol Hypertens 1997; 6: 333-348

11. Gerakis A, Hutchinson AJ, Apostolou T, Freemont AJ, Billis A. Biochemical markers for non invasive diagnosis of hyperparathyroid bone disease and adynamic bone in patients on haemodialysis. Nephrol Dial Transplant 1996; 11: 2430-2438

12. Wang M, Hercz G, Scherrard D, Maloney N, Segre G, Pei Y. Relationship between intact 1-84 parathyroid hormone and bone histomorphometric parameters in dialysis patients without aluminium toxicity. Am J Kidney Dis 1995; 26: $836-844$

13. Brossard J, Cloutier M, Roy L, Lepage R, Gascon Barré M, D'Amour P. Accumulation of a non (1-84) molecular form of parathyroid hormone detected by intact PTH assay in renal failure. Importance in the interpretation of PTH values. $J$ Clin Endocrinol Metab 1996; 81: 3923-3929

14. Hesch R, Busch U, Prokop M, Delling G, Rittinghaus EF. Increase of vertebral density by combination therapy with pulsatile 1-38hPTH and sequential addition of calcitonin nasal spray in osteoporotic patients. Calcif Tissue Int 1989; 44: $176-180$

15. Kim H, Chang K, Lee $\mathrm{T}$, Kwon J, Park S. Bone mineral density after renal transplantation. Transplant Proc 1998; 30: 3029-3030

16. Grotz WH, Mundinger FA, Rasenack $\mathbf{J}$ et al. Bone loss after kidney transplantation: a longitudinal study in 115 graft recipients. Nephrol Dial Transplant 1995; 10: 2096-2100

17. Torregrosa JV, Campistol JM, Montesinos M, Pons F, Martinez de Osaba MJ. Evolution of bone mineral density after renal transplantation: related factors. Nephrol Dial Transplant 1995; 10 [Suppl 6]: 111-113

18. Bryer $\mathrm{H}$, Isserow $\mathrm{J}$, Armstrong $\mathrm{E}$ et al. Azathioprine alone is bone sparing and does not alter cyclosporin A-induced osteopenia in the rat. J Bone Miner Res 1995; 10: 132-138

19. Wilmink J, Bras J, Surachno S, van Heyst J, van der Horst J. Bone repair in cyclosporine treated renal patients. Transplant Proc 1989; 21: 1492

20. Epstein S. Post-transplantation bone disease: the role of immunosuppressive agents and the skeleton. [Review]. $J$ Bone Miner Res 1996; 11: 1-7

21. Kelly P, Sambrook P, Eisman J. Potential protection by cyclosporine against glucocorticoid effects on bone. Lancet 1989, ii: 1388

22. Stewart P, Stern P. Cyclosporine A: correlation of immunosuppressive activity and inhibition of bone resorption. Calcif Tissue Int 1989; 45: 222-226

23. Mottet J, Horber F, Casez J, Descoeudres C, Jaeger P. Evidence for preservation of cortical bone mineral density in patients on continuous ambulatory peritoneal dialysis. $J$ Bone Miner Res 1996; 11: 96-104

24. Handelsman DJ. Hypothalamic-pituitary gonadal dysfunction in renal failure, dialysis and renal transplantation. Endocrinol Rev 1985; 6: 151-182

25. Torres A, Lorenzo V, Salido E. Calcium metabolism and skeletal problems after transplantation. J Am Soc Nephrol 2002; 13: $551-558$

Received for publication: 24.1 .00

Accepted in revised form: 14.2 .02 